\title{
Design of a Data-Driven Control System for Reference Model Design using Predicted Signals
}

\author{
Yuki Nakatani*, Takuya Kinoshita, Toru Yamamoto \\ Graduate School of Advanced Science and Engineering, Hiroshima University, 1-4-1 Kagamiyama, Higashi-Hiroshima \\ Higashi-Hiroshima-shi, Hiroshima-ken, 739-8527, Japan ${ }^{\dagger}$ \\ E-mail: \{nakatani-yuki, kinoshita-takuya, yama\}hiroshima-u.ac.jp \\ www.hiroshima-u.ac.jp
}

\begin{abstract}
In recent years, data-driven control schemes that do not require system identification have been actively studied. Generally, it is easy to give a reference model focusing only on the output response. In contrast, it is difficult to give a reference trajectory considering the input signals based on the controlled system's characteristics. Furthermore, it is necessary to consider the output signal and the input signal since there is a limit of the actuator performance in the control design of the actual machine. This paper proposes a data-driven control scheme that can predict the input/output response of an unknown system in offline using operating data. The effectiveness of the proposed scheme is numerically verified by a simulation example.
\end{abstract}

Keywords: Data-driven control, extended output, predicted data, off-line tuning.

\section{Introduction}

In industrial systems, data-driven control methods ${ }^{1,2}$ have been actively studied to achieve the desired control performance for the controlled system with unknown structure and parameters. These methods use only a set of experimental data to design a controller that realizes the desired reference output offline. However, the control system design should consider the input/output response because there is a limit of the actuator performance of the actual machine.

The ERIT (Estimated Response Iterative Tuning) method $^{3}$ has been proposed to predict the input/output response. However, this method can only be applied to two-degree-of-freedom (2DOF) control systems ${ }^{4}$. On the other hand, it is useful to design a controller to consider input signals in one-degree-of-freedom (1DOF) because there are also many 1DOF control systems in the industry. Therefore, in this paper, the new data-driven control scheme is proposed to design a 1DOF controller considering input signals for unknown structure system. The features of this scheme are as follows.

(i) 1DOF control system design that predicts input signals in advance, even for unknown controlled system

(ii) Adjusting the desired input/output response with one parameter

\section{Overview of the proposed scheme}

Fig. 1 shows an overview of the proposed data-driven control scheme. In the proposed scheme, the controller is designed by the following procedure.

(I) Obtaining the desired predicted data $\hat{y}^{*}(t)$ and $\hat{u}^{*}(t)$

(II) Constructing the reference model $G_{m}\left(z^{-1}\right)$ based on $\hat{y}^{*}(t)$ and $\hat{u}^{*}(t)$ obtained in (I)

(III) Designing a controller using the reference model $G_{m}\left(z^{-1}\right)$ obtained in (II)

The detailed procedure is explained in the next section. 


\section{Design of the controller}

\subsection{Generating predicted the data using the ERIT method}

In this section, the ERIT method ${ }^{3}$ is described to generate the predicted data. This method is theoretically only applicable to 2DOF control system ${ }^{4}$. Therefore, in the proposed scheme, the ERIT method is utilized for only predicting the input/output data in Fig. 2. Note that the controller is not designed by the ERIT method.

First, the initial output $y_{0}(t)$ is obtained in a $1 \mathrm{DOF}$ control system. In the case of the feed-forward controller $C_{F F}\left(z^{-1}\right)=0$ in Fig. 2, $y_{0}(t)$ is given as follows:

$$
y_{0}(t)=\frac{G\left(z^{-1}\right) C_{F B}\left(z^{-1}\right)}{1+G\left(z^{-1}\right) C_{F B}\left(z^{-1}\right)} r(t) .
$$

Then, extending to a $2 \mathrm{DOF}$ control system for predicting the data, the predicted output $\hat{y}(t)$ is defined as:

$$
\hat{y}(t)=\frac{G\left(z^{-1}\right) C_{F F}\left(z^{-1}\right)+G\left(z^{-1}\right) C_{F B}\left(z^{-1}\right)}{1+G\left(z^{-1}\right) C_{F B}\left(z^{-1}\right)} r(t) .
$$

However, system identification is required to obtain an exactly predicted output $\hat{y}(t)$ because Eq. (2) contains a controlled system $G\left(z^{-1}\right)$. Therefore, substituting Eq. (1) into Eq. (2) yields the following equation:

$$
\hat{y}(t)=\frac{C_{F F}\left(z^{-1}\right)}{C_{F B}\left(z^{-1}\right)} y_{0}(t)+y_{0}(t) .
$$

Consequently, the predicted data $\hat{y}(t)$ can be derived offline without $G\left(z^{-1}\right)$ from the initial data $y_{0}(t)$ by changing $C_{F F}\left(z^{-1}\right) . \hat{u}(t)$ can also be derived in the same procedure using $u_{0}(t)$ as follows:

$$
\hat{u}(t)=\frac{C_{F F}\left(z^{-1}\right)}{C_{F B}\left(z^{-1}\right)} u_{0}(t)+u_{0}(t) .
$$

From Eqs. (3) and (4), it is possible to obtain the predicted data $\hat{y}(t)$ and $\hat{u}(t)$ offline corresponding to the various $C_{F F}\left(z^{-1}\right)$ by using the initial data $y_{0}(t)$ and $u_{0}(t)$. Note that the $2 \mathrm{DOF}$ control system was only implemented virtually off-line to obtain the predicted data.

\subsection{Obtaining the desired predicted input/output data}

In this section, obtaining the desired predicted data $\hat{y}^{*}(t)$ and $\hat{u}^{*}(t)$ is described by using the predicted data $\hat{y}(t)$ and $\hat{u}(t)$. Specifically, $\hat{y}^{*}(t)$ and $\hat{u}^{*}(t)$ are calculated by solving the following minimization

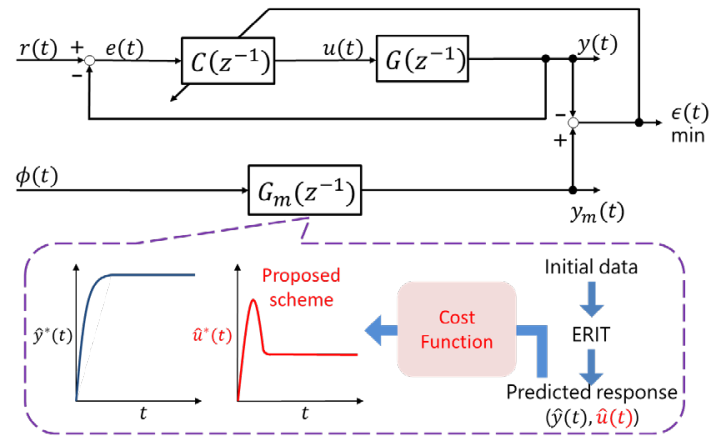

Fig. 1. Overview of the data-driven control system by the proposed scheme.

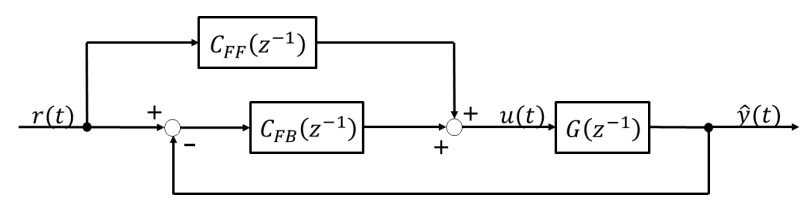

Fig. 2. 2DOF control system.

problem of the evaluation norm based on the ITAE (Integral of Time squared Absolute Error) method ${ }^{5}$ for $C_{F F}\left(z^{-1}\right)$ :

$$
J_{\text {des }}=\sum_{t=0}^{N} t|r(t)-\hat{y}(t)|+\lambda \sum_{t=0}^{N} t|\Delta \hat{u}(t)|,
$$

where $r(t)$ and $\Delta\left(:=1-z^{-1}\right)$ denote the reference signal and the difference operator.

Firstly, $r(t)-\hat{y}(t)$ in Eq. (5) shows the difference between the reference signal and the predicted output signal. The desired predicted response is obtained by reducing it. Secondly, $\Delta \hat{u}(t)=\hat{u}(t)-\hat{u}(t-1)$ in Eq. (5) represents the difference of input signal. Therefore, a small value of $\lambda$ results in a highly responsive response, while a large value of $\lambda$ results in a less responsive response by depending on the tunable parameter $\lambda$. Thus, the input/output response can be easily adjusted with $\lambda$. Additionally, it is easy for the user to select the optimal $\hat{y}^{*}(t)$ and $\hat{u}^{*}(t)$ by changing $\lambda$ because predicted data of unknown system can be calculated in the ERIT method.

The adjustment of $\lambda$ is currently a trial and error process. However, this process is very easy because it is only necessary to adjust $\lambda$ offline.

\subsection{Constructing $G_{m}\left(z^{-1}\right)$ based on the desired predicted data $\widehat{\boldsymbol{y}}^{*}(\boldsymbol{t})$}

In this section, constructing $G_{m}\left(z^{-1}\right)$ is described based on the desired predicted data $\hat{y}^{*}(t)$. The evaluation function for constructing $G_{m}\left(z^{-1}\right)$ is defined as follows: 


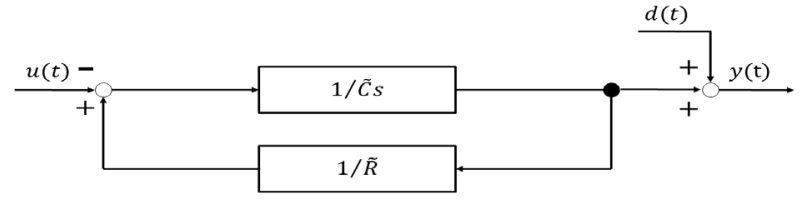

Fig. 3. Block diagram of a temperature control device.

$$
\begin{gathered}
J_{\text {ref }}=\frac{1}{N} \sum_{t=0}^{N}\left(\hat{y}^{*}(t)-y_{m}(t)\right)^{2} \\
y_{m}(t)=G_{m}\left(z^{-1}\right) r(t),
\end{gathered}
$$

where $N$ denotes the number of data. $G_{m}\left(z^{-1}\right)$ is constructed by minimizing the evaluation $J_{r e f}$.

\subsection{Design of a PID controller based on extended output}

In this section, a $1 \mathrm{DOF}$ controller $C\left(z^{-1}\right)$ is designed as an I-PD controller ${ }^{6}$ by using the reference model $G_{m}\left(z^{-1}\right)$ that considers the input response obtained in the previous section.

I-PD controller is given as follows:

$$
\Delta u(t)=K_{I} e(t)-K_{P} \Delta y(t)-K_{D} \Delta^{2} y(t),
$$

where $K_{P}, K_{I}$, and $K_{D}$ are the proportional gain, integral gain, and derivative gain respectively. In this paper, PID gains are tuned by using the extended output. In this method, $C\left(z^{-1}\right)$ is designed by minimizing the evaluation norm based on the property that the extended output $\phi(t)$ and the reference signal $r(t)$ are equal in Fig. 1. In other words, the evaluation norm is defined as:

$$
\begin{gathered}
J=\frac{1}{N} \sum_{t=0}^{N} \epsilon(t)^{2} \\
\epsilon(t)=G_{m}\left(z^{-1}\right) \phi(t)-y(t) .
\end{gathered}
$$

where $\epsilon(t)$ denotes the difference between the output $y(t)$ of the closed-loop transfer function and the output $G_{m}\left(z^{-1}\right) \phi(t)$ of the reference. $C\left(z^{-1}\right)$ can be designed by minimizing the evaluation $J$. For reasons of space, the details are omitted, refer to the PID control method based on extended output ${ }^{7}$.

\section{Numerical example}

\subsection{Controlled system}

The effectiveness of the proposed scheme is verified using the numerical simulation. The controlled system is the thermal experimental apparatus for simulating bag-
Table 1. Obtained parameters in the simulation.

\begin{tabular}{|c|c|c|}
\hline & $\lambda=1$ & $\lambda=35$ \\
\hline$\alpha_{1}$ & $2.23 \times 10^{-2}$ & $1.71 \times 10^{-4}$ \\
\hline$\alpha_{2}$ & 1 & 1 \\
\hline$\alpha_{3}$ & $-2.35 \times 10^{-2}$ & $-1.57 \times 10^{-2}$ \\
\hline$\alpha_{4}$ & $-4.07 \times 10^{-2}$ & $-9.75 \times 10^{-1}$ \\
\hline$\theta_{1}$ & $2.23 \times 10^{-4}$ & $1.72 \times 10^{-6}$ \\
\hline$\theta_{2}$ & $8.92 \times 10^{-4}$ & $6.89 \times 10^{-6}$ \\
\hline$\theta_{3}$ & 1 & 1 \\
\hline$\theta_{4}$ & -1.42 & -1.99 \\
\hline$\theta_{5}$ & $4.20 \times 10^{-1}$ & $9.93 \times 10^{-1}$ \\
\hline$K_{P}$ & $4.24 \times 10^{2}$ & 4.27 \\
\hline$K_{I}$ & $8.16 \times 10^{-1}$ & $5.60 \times 10^{-3}$ \\
\hline
\end{tabular}

and-bound welding owned by our laboratory. Fig. 3 shows the model of the system, and the coefficients were derived by system identification. The details are omitted due to space limitation.

Then, the proposed scheme is applied to the system in Fig. 3. The reference signal is $r(t)=100$, the sampling time is $\mathrm{T}_{\mathrm{S}}=0.02 \mathrm{~s}$, and the room temperature is $d(t)=$ $20\left[{ }^{\circ} \mathrm{C}\right]$. In this numerical example, I-P controller is used, and the proportional gain and integral gain are set as follows:

$$
K_{P}=1.5, K_{I}=1.0 \times 10^{-3} .
$$

Next, the feed-forward controller $C_{F F}^{*}\left(z^{-1}\right)$ was calculated by the proposed scheme as follows:

$$
C_{F F}^{*}\left(z^{-1}\right)=\frac{\alpha_{1}}{\alpha_{2}+\alpha_{3} z^{-1}+\alpha_{4} z^{-2}} .
$$

The order of the denominator was set to second order because the order which significantly affects the output response of controlled system is generally low order. Furthermore, the value of $\alpha_{i}(i=1,2,3,4)$ is determined to minimize $J_{\text {des }}$ in Eq. (5), for example, using the Matlab/Simulink

Ver.9.8.0.1396136(R2020a),

Optimization Toolbox 'fminsearch.m '.

Furthermore, $G_{m}\left(z^{-1}\right)$ was determined as follows:

$$
G_{m}\left(z^{-1}\right)=\frac{\theta_{1}+\theta_{2} z^{-1}}{\theta_{3}+\theta_{4} z^{-1}+\theta_{5} z^{-2}}
$$



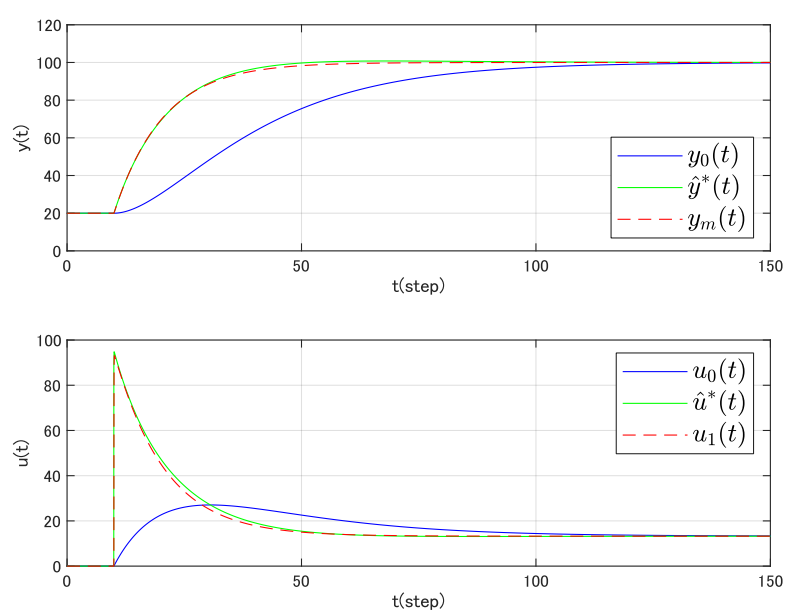

Fig. 4. Simulation results by applying the proposed scheme where $\lambda=1$.

where the value of $\theta_{i}(i=1,2,3,4,5)$ is determined by using the least-squares method to minimize $J_{\text {ref }}$ in Eq. (6).

In this numerical example, the results are compared by changing $\lambda$ in Eq. (5), and Table 1 shows the feedforward controller $C_{F F}^{*}\left(z^{-1}\right)$, the reference model $G_{m}\left(z^{-1}\right)$, the proportional gain, and the integral gain respectively in the cases of $\lambda=1,35$.

\subsection{Control result}

Figs. 4 and 5 show the initial data $y_{0}(t)$ and $u_{0}(t)$, the desired predicted data $\hat{y}^{*}(t)$ and $\hat{u}^{*}(t)$, and the results of implementing a 1DOF controller $y_{1}(t)$ and $u_{1}(t)$ in the cases of $\lambda=1,35$. The input-output response can be adjusted with one parameter according to $\lambda$ because the responsiveness is high because $\lambda=1$ in Fig. 4 , and the responsiveness is low because $\lambda=35$ in Fig. 5 . Furthermore, it is possible to design a controller that considers input signals because the input signals $\hat{u}^{*}(t)$ and $u_{1}(t)$ are almost identical in Figs. 4 and 5.

\section{Conclusion}

In this paper, the new data-driven control scheme has been proposed to design a 1DOF to consider input signal for unknown structure system. The effectiveness has been verified by the numerical example. It is possible to predict the appropriate amount of input signal to correspond the performance of the actuator by predicting the input in advance. In the future, the method how to
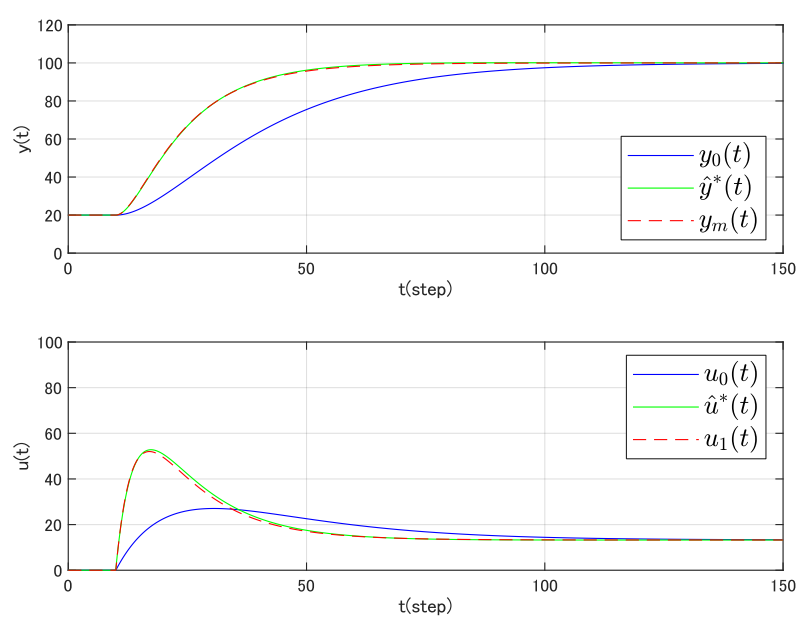

Fig. 5. Simulation results by applying the proposed scheme where $\lambda=35$.

choose $\lambda$ and the controller design considering the input saturation will be studied.

\section{References}

1. T. Yamamoto, O. Kaneko, S. Wakitani, T. Kinoshita, Y. Ohnishi, H. Kugemoto, K. Koiwai, Data-oriented PID control, Morikita publish, 2020.

2. T. Yamamoto, O. Kaneko, A Direct Design Method for Control Systems Based on Closed-Loop Data, Journal of the SICE 52(10), 2013, pp. 841-846

3. O, Kaneko, H. Nakamura, T. Ikezaki, A New Approach to Updating Feedforward Controllers in Two-Degree-ofFreedom Control Systems Estimated Response Iterative Tuning (ERIT) is proposed, Transactions of the SICE 54(12), 2018, pp. 857-864.

4. Y. Chida, Design Method of Two-Degree-of-Freedom Control System Using Characteristics of Feedback Controller, Transactions of the Institute of Electrical Engineers of Japan 117(4), 1997, pp.439-445

5. M. Masubuchi, Revised Basic Theory of Automatic Control, Corona Corporation, 2005, pp. 181-184

6. T. Kitamori, Principles and Design Methods of I-PD Control Systems (Special Issue on PID Control - Theory and Application), Transactions of SICE 54(12), 1998, pp. $7-17$

7. A, Yoichiro, S. Wakitani and T. Yamamoto, Design of an Augmented Output-Based Multiloop Self-Tuning PID Control System, Industrial \& Engineering Chemistry Research 58(26), 2019, pp. 11474-11484

(C) The 2021 International Conference on Artificial Life and Robotics (ICAROB2021), January 21 to 24, 2021 